\title{
Dinámica poblacional de la palma Euterpe oleracea (Arecaceae) en bosques inundables del Chocó, Pacífico colombiano
}

\author{
Diego A. Arango ${ }^{1}$, Álvaro J. Duque ${ }^{1}$ \& Edinson Muñoz ${ }^{2}$ \\ 1. Departamento de Ciencias Forestales, Universidad Nacional de Colombia, Sede Medellín, A.A. 1027 Medellín, \\ Colombia; daarango@unal.edu.co,ajduque@unal.edu.co \\ 2. Fundación Con-Vida, calle 45G 77AA-16, Medellín, Colombia; edimciro@gmail.com
}

Recibido 02-III-2009. Corregido 20-VIII-2009. Aceptado 21-IX-2009.

\begin{abstract}
Population dynamics of the palm Euterpe oleracea (Arecaceae) from flooded forests in Choco, Colombian Pacific. The palm Euterpe oleracea is a dominant and promising species in flood plains of the Atrato river, Choco region of Colombia. We assessed the population dynamics of this species through growth rates, mortality and recruitment patterns for a period of two and a half years. Dynamic rates were compared among mixed and pure flood plain palm forests. These forests types were associated to different flooding regimes. Trees and palms were thinned in a portion for each forest type, the rest was left undisturbed. We used projection matrices to follow population trends. Thinning increased the transition probability of smaller individuals, but decreased it for larger individuals, as is typical of light demanding species. Thinning also increased mortality rates in almost all size classes, but did not affect recruitment rates. Under natural conditions, the $E$. oleracea populations are in equilibrium in pure and mixed forests. Thinning increased population growth in both forest types, suggesting the role played by density-dependent processes on the population size of this species. Rev. Biol. Trop. 58 (1): 465-481. Epub 2010 March 01.
\end{abstract}

Key words: population dynamics, Euterpe oleracea, demography, matrix models, sustainability, Choco Biogeografico.

Las palmas son organismos vegetales conspicuos en los bosques tropicales y proveen gran variedad de bienes, tales como frutos, fibras y materiales de construcción, ampliamente utilizados en los trópicos por pobladores locales (Balick 1989, Bernal 1992, Duran \& Franco 1992, Borgtoft 1994, 1996, Vormisto 2002). Algunos productos alcanzan, incluso, los grandes mercados mundiales. Adicionalmente, las palmas desempeñan un papel relevante en la dinámica y funcionamiento del bosque, debido al impacto de la caída de sus grandes hojas sobre individuos del sotobosque y su importancia como fuente de alimento para polinizadores y consumidores de semillas y frutos (Durán \& Franco 1992).

El Pacífico colombiano, ubicado en el Chocó Biogeográfico, es una de las regiones con mayor prioridad de conservación debido principalmente a su alta diversidad, fragilidad y endemismo (Mittermeier et al. 1999). Esta zona, caracterizada por un muy bajo ingreso per capita, requiere modelos económicos con énfasis en la sostenibilidad, que difieran de la explotación maderera intensiva que ha propiciado parte del sustento de las comunidades locales durante décadas (González 1995, del Valle 1996). Por lo anterior, la identificación de productos no maderables asociados con aprovechamientos sostenibles de poblaciones estables o en crecimiento se convierte en una opción prioritaria (Vásquez \& Gentry 1989, Pinard 1993).

La demografía es el área de la ecología que estudia la dinámica de poblaciones biológicas con el propósito de caracterizar sus cambios en 
términos de nacimientos, muertes y transiciones entre diferentes estados, tamaños o edades (Silva et al. 1999). Las matrices de transición por estados (Lefkovitch 1965) se adaptan fácilmente para modelar la dinámica poblacional de plantas; en algunos casos, el tamaño o estado de desarrollo son determinantes más importantes del comportamiento demográfico que la edad cronológica (Kohyama 1993, Condit et al. 1998). Estos modelos matriciales constituyen una herramienta óptima para describir la historia de vida de una especie, analizar la persistencia de una población y comparar la dinámica entre diferentes poblaciones (Silvertown et al. 1993, Caswell 2001). En este campo, la mayor parte de las hipótesis se ha centrado en analizar si los sistemas biológicos están o no en equilibrio (MacArthur \& Wilson 1967, Connell 1978, Muller-Landau et al. 2006, Coomes \& Allen 2007), fundamentos que permiten determinar el potencial de uso y aprovechamiento de recursos naturales en bosques húmedos tropicales, sin afectar su sostenibilidad (Svenning \& Balslev 1997).

En el presente estudio se determinaron algunos parámetros demográficos que caracterizan dos poblaciones de Euterpe oleracea Mart., sometidas a diferentes niveles de inundación, en el valle medio del río Atrato, Pacífico colombiano. E. oleracea ha sido reconocida como especie típica de bosques inundables (Galeano \& Bernal 1987), por lo que se asocia con una dominancia diferencial relacionada con el régimen hídrico. De manera complementaria, se evaluó el efecto de la competición por luz sobre los patrones demográficos a través de una intervención antrópica controlada, consistente en la reducción del nivel de ocupación del componente arbóreo del bosque.

Las hipótesis establecidas aquí fueron: $i$ ) bajo condiciones naturales, E. oleracea presenta mayor tasa de crecimiento poblacional cuando está sometida a mayores regímenes de inundación, y ii) la apertura del dosel por medio de la eliminación de algunos individuos, lo que incrementa la disponibilidad lumínica, promueve el aumento de las tasas de crecimiento poblacional, comparadas con las condiciones naturales. Con base en estos resultados, así como en cambios simulados sobre los parámetros de crecimiento, sobrevivencia y fertilidad, se evaluaron las posibilidades de aprovechamiento de E. oleracea, de la cual es posible extraer su meristema apical o palmito.

\section{MATERIALES Y MÉTODOS}

Sitio de estudio: Se localizó en el Municipio de Vigía del Fuerte (Antioquia, Colombia), en el valle medio del río Atrato, dentro de la zona de vida bosque muy húmedo tropical (bmh-T), con temperatura media de $28^{\circ} \mathrm{C}$, precipitación anual de 5 000-7 000mm (Espinal 1992, Eslava 1993, Pinto-Escobar 1993) y altitud aproximada de $45 \mathrm{msnm}$.

Los suelos se clasifican como Entisoles e Inceptisoles, y presentan fertilidad moderada a baja, $\mathrm{pH}$ de 4.2-5.2 y altos contenidos de materia orgánica (Cortés 1993). Los suelos de los planos inundables están sometidos a inundaciones periódicas, de magnitud y duración acorde con la posición fisiográfica, donde se forman complejos de humedales que posibilitan el desarrollo de comunidades vegetales diferenciables dominadas por pocas especies (Universidad Nacional de Colombia 1996, 1999).

Los datos demográficos fueron colectados en dos asociaciones vegetales dominadas por $E$. oleracea, conocida localmente como murrapo, sobre la margen oriental del río Atrato, cerca de la confluencia con el río Murrí. El murrapal mixto (bloque I, 6³4'52” N-7652'10” W) se localiza sobre el plano más alto; allí, el bosque es estacionalmente inundable y, además, se da la presencia significativa de otras palmas (Oenocarpus bataua, O. mapora, Mauritiella macroclada y Manicaria saccifera).

El murrapal puro (bloque II, 6 31 '58' $\mathrm{N}-76^{\circ} 50^{\prime} 40^{\prime}$ 'W) se asienta en un plano inferior, en una zona de depresión; el bosque permanece inundado y los sustratos más firmes están constituidos por raíces epigeas de E. oleracea y material vegetal diverso que se acumula en sus alrededores. Pocas especies arbóreas alcanzan niveles apreciables de ocupación: Pterocarpus officinalis (Fabaceae), 
Pentaclethra macroloba (Mimosaceae) y Grias cauliflora (Lecythidaceae).

Especie de estudio: E. oleracea es una palma cespitosa que forma grupos de muchos tallos (hasta 98, observación de campo), los cuales son soportados por una masa de raíces epigeas rojas con neumatóforos de colores pardo a crema; alcanza alturas hasta de $25 \mathrm{~m}$ y diámetros hasta de $18 \mathrm{~cm}$. Sus hojas son pinnadas, hasta 12 , de $1-4 \mathrm{~m}$ de longitud y $0.75-1.10 \mathrm{~m}$ de ancho; las pinnas son numerosas y péndulas. Las inflorescencias son ramificadas, con longitudes entre 80 y $110 \mathrm{~cm}$; las flores se presentan como triadas o estaminadas solitarias. Los frutos son globosos, con diámetro medio de $13 \mathrm{~mm}$ y epicarpio verde cuando inmaduro a moradonegro en la madurez (Galeano \& Bernal 1987, Henderson et al. 1995).

La especie se encuentra en dos regiones del trópico americano: $i$ ) en la costa pacífica colombo-ecuatoriana, y ii) sobre la costa atlántica, desde el noreste de Venezuela hasta el norte de Brasil; en Colombia, además, se observa en los valles medios de los ríos Atrato y Magdalena. Crece en zonas húmedas a pluviales, por debajo de $200 \mathrm{msnm}$, sobre suelos que permanecen inundados gran parte del año. Constituye formaciones homogéneas, especialmente en zonas bajas de los ríos (Galeano \& Bernal 1987, Henderson et al. 1995, Restrepo 1996).

Los frutos de E. oleracea se utilizan para preparar diversos tipos de bebidas, las brácteas se emplean para producir papel y concentrados para animales domésticos, y los tallos también parecen presentar características adecuadas para la fabricación de papel. En América tropical, el aprovechamiento del meristema apical o corazón de la yema terminal, conocido como palmito, es el principal uso (Galeano \& Bernal 1987, Gonçalves \& Kageyama 1994, Restrepo 1996, Universidad Nacional de Colombia 2000).

Unidades de muestreo: Ambos bloques fueron establecidos y evaluados en abril-mayo de 1997, cada uno conformado por 12 parcelas de $500 \mathrm{~m}^{2}(25 \times 20 \mathrm{~m})$, con disposición de $6 \times 2$ e incluyendo corredores de separación entre parcelas de 10m (Fig. 1). En cada parcela, todos los individuos del componente arbóreo con diámetro normal, a $1.3 \mathrm{~m}$ del suelo $(D)$ mayor o igual a $10 \mathrm{~cm}$ fueron marcados, medidos e identificados; para E. oleracea, todas las cepas fueron observadas y se registró el número total de tallos o estipes por categorías de altura: 1 (0.0-1.3m), 2 (1.3-2.5m), 3 (2.5-5.0m), 4 (5.0$8.0 \mathrm{~m}), 5(8.0-12.0 \mathrm{~m})$ y 6 ( $12 \mathrm{~m}$ en adelante). La altura hasta la base de la hoja guía o flecha $\left(H_{g}\right)$ se escogió como variable de referencia ya que es menos susceptible a alteraciones producidas por viento u otros factores.

En cada cepa y por cada categoría de altura, un estipe fue seleccionado al azar, marcado y medido su $D$, así como $H_{g}$. Estos estipes fueron evaluados en censos posteriores, definiendo su

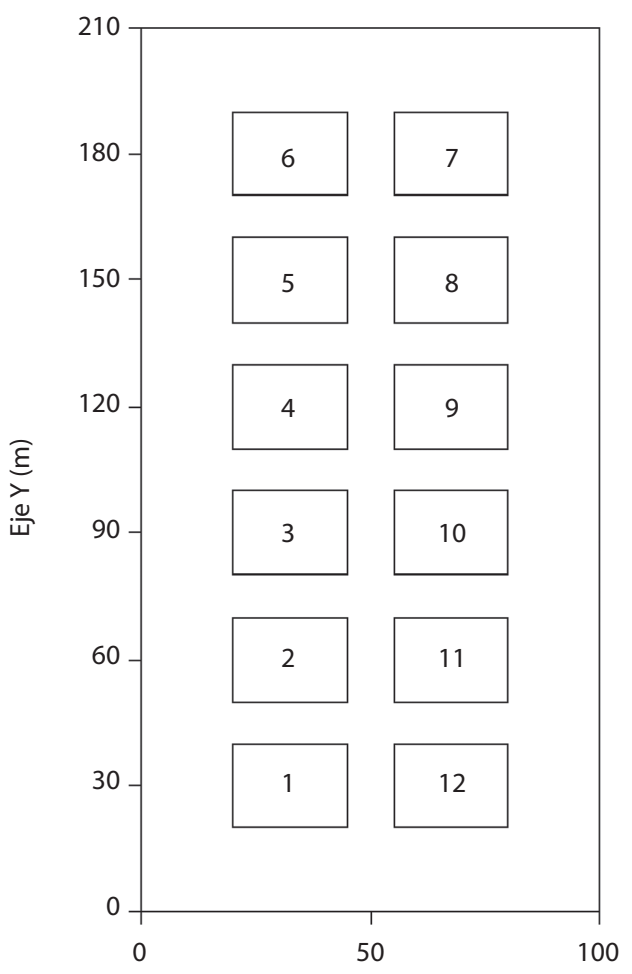

Eje X (m)

Fig. 1. Diseño del bloque y distribución de parcelas.

Fig. 1. Block design and plot distribution. 
condición de vivo o muerto, al igual que la densidad de estipes por categoría de altura por cepa en abril-1998 (ambos bloques), octubre-1999 (bloque I) y febrero-2000 (bloque II).

Cuando los intervalos de las clases de tamaño son muy pequeños, los estipes pueden avanzar dos o tres categorías por encima de la que los contiene inicialmente (Huenneke \& Marks 1987). Sin embargo, en este estudio, muy pocos tallos avanzaron más de una clase de tamaño, por lo que las categorías definidas no se consideraron pequeñas. Igualmente, si las clases son muy grandes, un estipe que recién ingresa a una de ellas requeriría muchos años para alcanzar la categoría inmediatamente superior (Huenneke \& Marks 1987); esto tampoco ocurrió, con base en las tasas de transición establecidas.

Tratamientos relacionados con la ocupación del sitio: Dos tratamientos fueron implementados en cada bloque: un control o testigo (parcelas 1, 3, 9 y 12 del murrapal mixto, y $5,6,8$ y 11 del murrapal puro, seleccionadas aleatoriamente) y uno intervenido (las ocho parcelas restantes por bloque). La intervención consistió en un aclareo por lo alto, tanto de individuos arbóreos como palmas, para disminuir la ocupación del bosque (expresada a través del área basal), situación que podría ocurrir si las comunidades dominadas por $E$. oleracea fueran objeto de aprovechamiento para la comercialización de palmito. El aclareo fue controlado, de forma que no se afectaran las parcelas control o testigo; en el murrapal mixto, la corta disminuyó el área basal de las parcelas afectadas a $45-66 \%$ (promedio 52\%) de la correspondiente a las parcelas testigo; en el murrapal puro, el resultado fue $43-79 \%$ (promedio 62\%) comparado con el área basal del testigo.

Determinación de tasas de fertilidad: La mayor parte de los nuevos estipes de E. oleracea proviene de la regeneración vegetativa o asexual. Éstos emergen alrededor o sobre la corona de raíces de cada cepa; sin embargo, no es posible identificar cuál estipe individual es el padre de un nuevo retoño (ramet). Por lo anterior, para cuantificar la producción de retoños por estipe, se modificó la propuesta de Huenneke \& Marks (1987). Así, el número de retoños producido por una cepa durante el intervalo de medición fue dividido por la suma de $H_{g}$ de los estipes que la componen (registrados al inicio del intervalo). Luego, esta tasa de producción de retoños (por metro de altura) fue multiplicada por el punto medio de cada clase de tamaño (categorías 1-6), para calcular la producción media de retoños $\left(S_{j}\right)$ por individuo de la clase de tamaño $j$. En este caso particular, la categoría de altura 1 sí presentó una tasa de producción de retoños diferente de 0 (respaldada por observaciones de campo).

Por otro lado, durante los censos de remedición, cada nueva plántula o individuo proveniente de una semilla (genet, regeneración sexual) de E. oleracea con altura superior a $50 \mathrm{~cm}$ fue considerada como una cepa; por tanto, fue marcada y se le midió el diámetro medio del tallo $(\mathrm{cm})$, así como $H_{g}$. La tasa de fertilidad $\left(F_{j}\right.$, para $j=5$ y 6$)$ fue calculada como la relación entre nuevas cepas y el número total de estipes de las categorías 5 y 6 , ya que éstos están en etapa reproductiva.

\section{Construcción de matrices de transición:}

Se conformaron cuatro matrices de transición, una por cada tratamiento en cada murrapal. Las probabilidades de transición fueron estimadas como la proporción de estipes que se movieron desde una clase de tamaño $\left(H_{g}\right)$ hacia otra $\left(p_{i, j}\right.$, proporción de estipes que pasan de la categoría de altura $i$ a la $j$ ). Las tasas de estipes que permanecieron en la misma categoría de tamaño a través del intervalo $\left(p_{i, j}\right.$, con $\left.i=j\right)$ conformaron los elementos de la diagonal de la matriz de transición. Durante el tiempo de medición (murrapal mixto: 2.39 años, murrapal puro: 2.79 años), sólo cinco estipes de E. oleracea $(0.38 \%)$ avanzaron más de una categoría de altura; por lo tanto, todo $p_{i, j}$ fue diferente de 0 si $j=i, j=1$ o $j=i+1$; fue 0 o un valor cercano si $j=i+2$; y fue 0 en los demás casos (Cuadro 1 ).

En cada situación, la matriz de transición resultante $A$ se utilizó para modelar los 
CUADRO 1

Estructura de la matriz de transición de E. oleracea con seis categorías de altura

TABLE 1

Structure of stage projection matrix for E. oleracea with six height sizes

$\begin{array}{cccccc}p_{1,1}+S_{1} & S_{2} & S_{3} & S_{4} & S_{5}+F_{5} & S_{6}+F_{6} \\ p_{1,2} & p_{2,2} & 0 & 0 & 0 & 0 \\ p_{1,3} & p_{2,3} & p_{3,3} & 0 & 0 & 0 \\ 0 & p_{2,4} & p_{3,4} & p_{4,4} & 0 & 0 \\ 0 & 0 & p_{3,5} & p_{4,5} & p_{5,5} & 0 \\ 0 & 0 & 0 & p_{4,6} & p_{5,6} & p_{6,6}\end{array}$

$p_{i, j}$ : probabilidad de transición de la clase de tamaño $i$ a $j, S_{j}$ : tasa de producción de retoños por estipe de la clase $j, F_{j}$ : tasa de producción de nuevas cepas por estipe de la clase $j$.

$p_{i, j}:$ transition probabilities; $S_{j}$ : ramets production rates by stem, $F_{j}$ : genets production rates by stem.

cambios de la densidad poblacional, a partir de la ecuación:

$$
N_{t+1}=A N_{t}
$$

donde $N_{t}$ es un vector de seis elementos que representa el número de estipes de cada categoría de altura en el tiempo $t$. Las tasas de transición no fueron alteradas y el vector de tamaño poblacional $N_{0}$ (densidad inicial) fue multiplicado iterativamente por $A$ hasta que la población alcanzara eventualmente un estado estable (de Kroon et al. 1986, van Groenendael et al. 1988, Caswell 2001, Vandermeer \& Goldberg 2003), tal que:

$$
\lambda N_{t}=A N_{t}
$$

La forma de la matriz poblacional $A$ permite la existencia de un valor no negativo de lambda $(\lambda)$ mayor que cualquier otro (valor propio dominante o eigenvalue dominante). Los valores de $\lambda$ reflejaron la tasa de crecimiento poblacional (de Kroon et al. 1986, van Groenendael et al. 1988, Caswell 2001); cuando $\lambda=1$, el tamaño total de la población no cambió; si $\lambda>1$, la población se estaba incrementando; si $\lambda<1$, la población estaba en decrecimiento. En términos demográficos, $\lambda=e^{r}$, donde $r$ es la tasa intrínseca de incremento natural (Caswell 2001, Vandermeer \&
Goldberg 2003). Los intervalos de confianza al $95 \%$ para cada $\lambda$ se obtuvieron mediante un procedimiento bootstrap a partir de 1000 simulaciones. Los elementos $p_{i, j}$ de cada matriz $A$ simulada fueron determinados mediante la selección aleatoria, con reemplazamiento, de los registros de campo que permitieron calcular $\operatorname{los} p_{i, j}$ de la matriz $A$ real.

El vector propio o eigenvector $(u)$ asociado a cada $\lambda$, con todos sus elementos reales no negativos, representó la distribución del estado estable por clases de tamaño. Si la ecuación 2 es verdadera, $N_{t}$ es reemplazado por $u$ y se genera una nueva relación, tal que (Caswell 2001, Vandermeer \& Goldberg 2003):

$$
\lambda u=A u
$$

Si la ecuación 3 es correcta, puede obtenerse un nuevo vector que representa la distribución del valor reproductivo ( $v$ ), así (Caswell 2001, Vandermeer \& Goldberg 2003):

$$
\lambda v=A^{T} v, \quad \text { (4) } \quad \text { ó } \quad \lambda v^{T}=v^{T} A
$$

donde $A^{T}$ es la matriz transpuesta de $A$ y $v^{T}$ es el vector traspuesto de $v$. El valor reproductivo es una expresión de la contribución de una clase de tamaño a todas las demás clases (Vandermeer \& Goldberg 2003). 
Matrices de sensibilidad y elasticidad: Los efectos causados por variaciones en las tasas de fertilidad y en las probabilidades de transición sobre la tasa de crecimiento poblacional fueron modelados (Hartshorn 1975). El cambio en $\lambda$ generado por estas alteraciones fue presentado en una matriz de sensibilidad; $s_{i, j}($ elementos de la matriz $S$ ) se obtuvo con base en $u$ y $v$ :

$$
s_{i, j}=\frac{v_{i} u_{j}}{(v u)}
$$

donde el denominador corresponde al producto escalar de ambos vectores (Pinard 1993, van Groenendael et al. 1988, Caswell 2001). Cada $s_{i, j}$ representó el cambio absoluto en $\lambda$ asociado con una alteración infinitesimal en $p_{i, j}$ de la matriz A (de Kroon et al. 1986).

La $p_{i, j}$ con mayor impacto sobre los $\lambda$ de los cuatro escenarios fue objeto de análisis, asignando valores a esta posición entre 0 y un valor $Z$ generado así:

$$
Z=1-\sum_{k=1}^{6} p_{i, k}, \mathrm{k} \neq \mathrm{j}
$$

A cada valor asignado de $p_{i, j}$, dejando las demás probabilidades de transición constantes, se determinó su respectivo $\lambda$. Estos pares ordenados fueron graficados para observar los efectos de tales cambios en las tasas de crecimiento poblacional.

El análisis de elasticidad evita algunos problemas de escala que conlleva la sensibilidad, ya que mientras $p_{i, j}$ oscila entre 0 y 1 , $F_{j}$ o $S_{j}$ pueden ser superiores a 1 . Dentro de la matriz de elasticidad $E$, cada $e_{i, j}$ representó la contribución proporcional de un cambio en $p_{i, j}$ sobre $\lambda$ (Vandermeer \& Goldberg 2003). El cálculo de $e_{i, j}$ se basó en la ecuación (de Kroon et al. 1986, Pinard 1993, Caswell 2001):

$$
e_{i, j}=\frac{p_{i, j}}{\lambda} \frac{v_{i} u_{j}}{(v u)}
$$

La aplicación PopTools 2.7.5 (sep. 25-2006, CSIRO Sustainable Ecosystems, Australia), instalada en el software Microsoft Excel 2002, fue utilizada para obtener tasas de crecimiento poblacional, distribuciones del estado estable y de los valores reproductivos, y matrices de sensibilidad y elasticidad.

\section{RESULTADOS}

En el murrapal mixto, se registraron 117 cepas de E. oleracea durante el censo inicial, 52 en el tratamiento testigo (área de muestreo: $\left.2000 \mathrm{~m}^{2}\right)$ y 65 en el tratamiento intervenido $\left(4000 \mathrm{~m}^{2}\right)$. En el murrapal puro se observaron 241 cepas, 81 en el testigo y 160 en el intervenido. Los vectores que expresan la densidad inicial por categoría de altura y por tratamiento se presentan en el Cuadro 2.

Fertilidad: Las tasas de fertilidad sexual $\left(F_{j}\right)$ fueron muy bajas, incluso no se registraron cepas nuevas en el murrapal mixto testigo. Los valores determinados para esta variable fueron: murrapal mixto intervenido: 0.0079 renuevos por individuo (categorías 5 y 6), murrapal puro testigo: 0.0069 e intervenido: 0.0185 . Las tasas relacionadas con la fertilidad asexual (propagación vegetativa o clonal), expresadas como retoños producidos por individuo de una clase de tamaño $j$ se detallan en el Cuadro 3.

Tasas de crecimiento poblacional y estado estable: Las probabilidades de transición variaron entre tipos de bosque y entre tratamientos (Cuadro 4). En el murrapal mixto testigo, la población se encontró en equilibrio $(\lambda=1, p>0.05$, rango: 0.979-1.217), a diferencia del crecimiento poblacional positivo registrado en el intervenido $(\lambda>1, p<0.05$, rango: 1.062$1.211)$; no obstante, los vectores que expresaron la distribución de la estructura estable $\mathrm{y}$ el valor reproductivo fueron similares entre tratamientos (Cuadro 5, Figs. 2, 3).

En el murrapal puro también se encontraron resultados opuestos; la tasa de crecimiento del testigo mostró un comportamiento poblacional orientado al equilibrio $(\lambda=1, \mathrm{p}>0.05$, rango: 0.893-1.033), mientras que en el intervenido fue positiva $(\lambda>1, \mathrm{p}<0.05$, rango: 1.047-1.162). 
CUADRO 2

Densidad total de tallos de E. oleracea por categoría de altura y tratamiento

TABLE 2

Total stem density of $\mathrm{E}$. oleracea by height size and management regime

\begin{tabular}{ccccc} 
Categoría & \multicolumn{2}{c}{ Murrapal mixto } & \multicolumn{2}{c}{ Murrapal puro } \\
de altura & Testigo & Intervenido & Testigo & Intervenido \\
1 & 590 & 749 & 768 & 2082 \\
2 & 77 & 55 & 98 & 224 \\
3 & 49 & 58 & 105 & 229 \\
4 & 31 & 49 & 67 & 128 \\
5 & 33 & 46 & 58 & 88 \\
6 & 69 & 80 & 233 & 183
\end{tabular}

CUADRO 3

Tasas de fertilidad asexual por categoría de altura (retoños producidos por individuo de la clase de tamaño j)

TABLE 3

Non-sexual fecundity rates by height size (number of ramets produced by an individual of the height size j)

\begin{tabular}{lcccccc}
\multicolumn{1}{c}{ Categoría } & 1 & 2 & 3 & 4 & 5 & 6 \\
Murrapal mixto testigo & 0.1045 & 0.3061 & 0.6034 & 1.0454 & 1.6078 & 2.4274 \\
Murrapal mixto intervenido & 0.1123 & 0.3290 & 0.6485 & 1.1234 & 1.7279 & 2.6086 \\
Murrapal puro testigo & 0.0581 & 0.1702 & 0.3355 & 0.5813 & 0.8940 & 1.6089 \\
Murrapal puro intervenido & 0.1301 & 0.3812 & 0.7514 & 1.3017 & 2.0020 & 3.6028
\end{tabular}

CUADRO 4

Matrices de probabilidades de transición para las poblaciones de E. oleracea

TABLE 4

Stage projection matrices of E. oleracea populations

\begin{tabular}{|c|c|c|c|c|c|}
\hline \multicolumn{3}{|c|}{ Murrapal mixto testigo } & \multirow{2}{*}{$\begin{array}{c}N=205 \\
1.045\end{array}$} & \multicolumn{2}{|l|}{$t=2.386$} \\
\hline 0.889 & 0.306 & 0.603 & & 1.608 & 2.427 \\
\hline 0.059 & 0.513 & - & - & - & - \\
\hline- & 0.333 & 0.633 & - & - & - \\
\hline- & - & 0.300 & 0.417 & - & - \\
\hline- & - & - & 0.500 & 0.348 & - \\
\hline- & - & - & 0.042 & 0.609 & 0.947 \\
\hline \multicolumn{3}{|c|}{ Murrapal mixto intervenido } & $N=231$ & $t=2.386$ & \\
\hline 0.690 & 0.329 & 0.648 & 1.123 & 1.736 & 2.617 \\
\hline 0.109 & 0.375 & - & - & - & - \\
\hline- & 0.375 & 0.634 & - & - & - \\
\hline- & - & 0.317 & 0.441 & - & - \\
\hline- & - & - & 0.500 & 0.625 & - \\
\hline- & - & - & - & 0.313 & 0.964 \\
\hline
\end{tabular}


CUADRO 4 (Continuación)

Matrices de probabilidades de transición para las poblaciones de E. oleracea

TABLE 4 (Continued)

Stage projection matrices of E. oleracea populations

$\begin{array}{cccccc} & \text { Murrapal puro testigo } & & N=323 & t=2.791 \\ 0.443 & 0.170 & 0.336 & 0.581 & 0.901 & 1.616 \\ 0.051 & 0.400 & - & - & - & - \\ - & 0.400 & 0.519 & - & - & - \\ - & - & 0.296 & 0.425 & - & - \\ - & - & 0.019 & 0.500 & 0.447 & 0.890 \\ - & - & - & 0.025 & 0.447 & 3.621 \\ 0.396 & \text { Murrapal puro intervenido } & & N=553 & t=2.791 & - \\ 0.119 & 0.381 & 0.751 & 1.302 & 2.020 & - \\ 0.007 & 0.216 & - & - & - & - \\ - & 0.495 & 0.409 & - & - & - \\ - & - & 0.487 & 0.452 & 0.644 & 0.894\end{array}$

$N$ : número de estipes evaluados, $t$ : tiempo entre censos.

$N$ : number of stems assessed, $t$ : time between censuses.

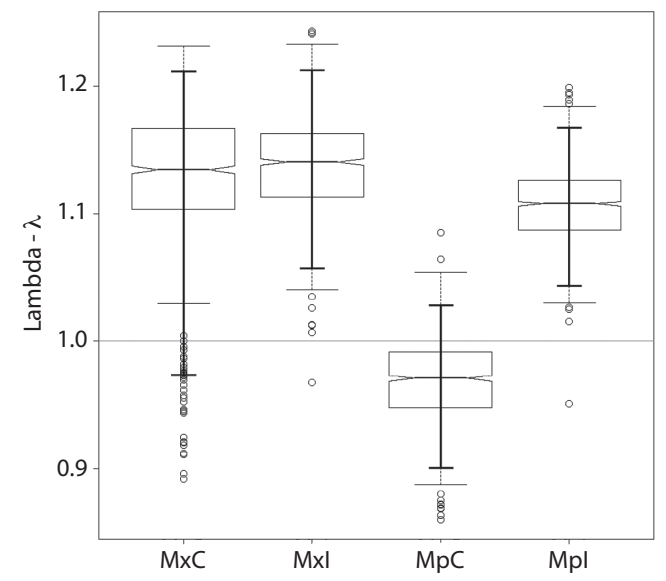

Fig. 2. Tasas de crecimiento poblacional $(\lambda)$ de las poblaciones de E. oleracea (MxC: murrapal mixto control o testigo, MxI: murrapal mixto intervenido, MpC: murrapal puro control o testigo, MpI: murrapal puro intervenido).

Fig. 2. Population growth rates $(\lambda)$ of E. oleracea populations (MxC: mixed murrapal-control, MxI: mixed murrapal-thinned, $\mathrm{MpC}$ : pure murrapal-control, $\mathrm{MpI}$ : pure murrapal-thinned).
Por otro lado, los vectores indicadores de la estructura estable también presentaron algunas particularidades, comparados entre sí y con los resultantes para el murrapal mixto. La distribución de la estructura estable del murrapal puro testigo fue comparable con la de ambos tratamientos en el murrapal mixto, excepto en $u_{6}(0.172 v s$. 0.044-0.056), equivalente al triple. En el murrapal puro intervenido ocurrió una disminución entre $u_{1}$ y $u_{2}$ considerablemente menor que la encontrada en los demás tratamientos (0.178 vs. 0.600$0.724)$; entre $u_{2}$ y $u_{3}$ continuó el descenso proporcional, lo que no ocurrió en el testigo, ni en el murrapal mixto. Los valores reproductivos presentaron tendencias homogéneas, siendo más altos en las mayores clases de tamaño (Cuadro 5, Figs. 2, 3).

Análisis de sensibilidad: Los mayores valores de $s_{i, j}$ en las matrices de sensibilidad de los tratamientos del murrapal mixto 


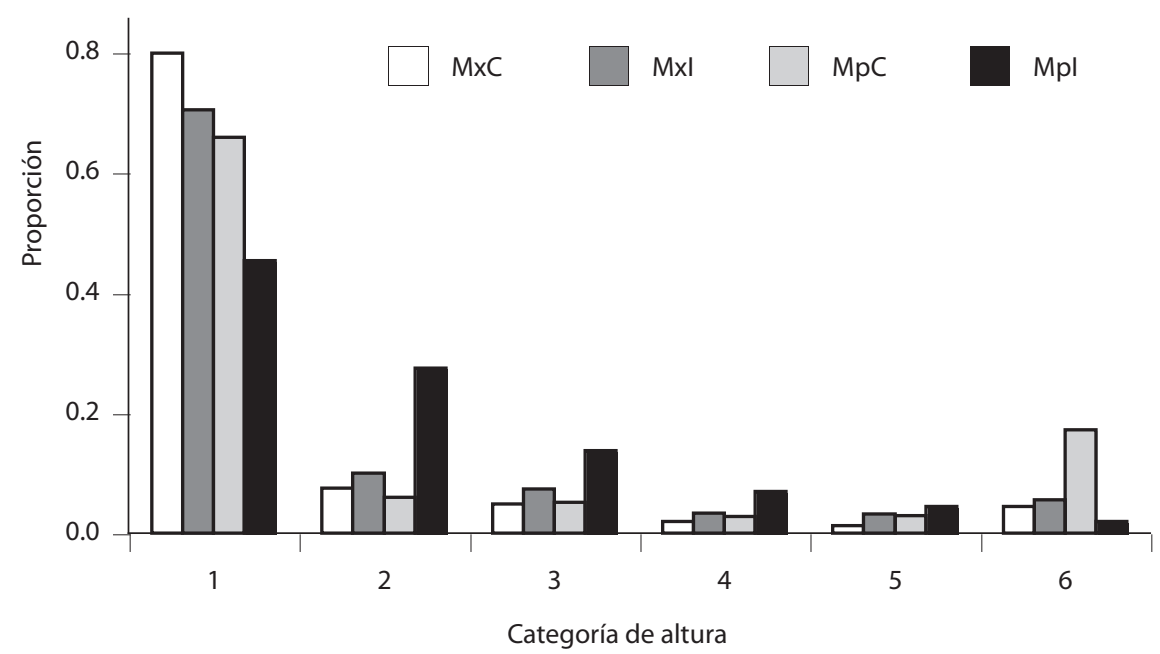

Fig. 3. Distribución de la estructura estable $\left(u_{j}\right)$ en las poblaciones de E. oleracea (MxC: murrapal mixto testigo, MxI: murrapal mixto intervenido, $\mathrm{MpC}$ : murrapal puro testigo, $\mathrm{MpI}$ : murrapal puro intervenido).

Fig. 3. Stable structure distribution $\left(u_{j}\right)$ of E. oleracea populations (MxC: mixed murrapal-control, MxI: mixed murrapalthinned, MpC: pure murrapal-control, $\mathrm{MpI}$ : pure murrapal-thinned).

\section{CUADRO 5}

Tasas de crecimiento poblacional, distribución de la estructura estable y valor reproductivo para $\mathrm{E}$. oleracea

TABLE 5

Population growth rates, stable structure distribution, and reproductive value of $\mathrm{E}$. oleracea

\begin{tabular}{|c|c|c|c|c|c|c|}
\hline \multicolumn{7}{|c|}{ Murrapal mixto testigo } \\
\hline$\lambda=$ & 1.142 & & & & & \\
\hline$u^{T}=$ & 0.799 & 0.075 & 0.049 & 0.020 & 0.013 & 0.044 \\
\hline$v^{T}=$ & 0.021 & 0.092 & 0.154 & 0.217 & 0.248 & 0.267 \\
\hline \multicolumn{7}{|c|}{ Murrapal mixto intervenido } \\
\hline$\lambda=$ & 1.144 & & & & & \\
\hline$u^{T}=$ & 0.705 & 0.100 & 0.074 & 0.033 & 0.032 & 0.056 \\
\hline$v^{T}=$ & 0.020 & 0.083 & 0.154 & 0.206 & 0.244 & 0.293 \\
\hline \multicolumn{7}{|c|}{ Murrapal puro testigo } \\
\hline$\lambda=$ & 0.970 & & & & & \\
\hline$u^{T}=$ & 0.659 & 0.059 & 0.052 & 0.028 & 0.029 & 0.172 \\
\hline$v^{T}=$ & 0.012 & 0.122 & 0.169 & 0.231 & 0.226 & 0.240 \\
\hline \multicolumn{7}{|c|}{ Murrapal puro intervenido } \\
\hline$\lambda=$ & 1.107 & & & & & \\
\hline$u^{T}=$ & 0.453 & 0.275 & 0.138 & 0.070 & 0.044 & 0.020 \\
\hline$v^{T}=$ & 0.050 & 0.068 & 0.127 & 0.182 & 0.228 & 0.344 \\
\hline
\end{tabular}

$\lambda$ : tasa de crecimiento poblacional, $u^{T}$ : vector transpuesto de distribución de la estructura estable, $v^{T}$ : vector transpuesto del valor reproductivo.

$\lambda$ : population growth rate, $u^{T}$ : transpose vector of stable structure distribution, $v^{T}$ : transpose vector of reproductive value. 
correspondieron a las probabilidades de crecimiento (avance a la clase de tamaño superior) de las tres categorías inferiores de altura y a las de permanencia en la clase superior $\left(s_{6,6}\right)$. En ambos casos, $s_{1,2}$ fue el registro más alto (Cuadro 6).

Respecto al murrapal puro, los tratamientos no fueron tan semejantes; la matriz de sensibilidad del testigo arrojó valores muy altos para la probabilidad de crecimiento de la clase de tamaño $1 \mathrm{y}$ la de permanencia de la clase 6 ; en el tratamiento intervenido, se destacaron los índices alcanzados por $s_{1,2}$ y $s_{1,3}$ (probabilidades de crecimiento de la clase 1 a las 2 y 3 , respectivamente).

El parámetro $p_{1,2}$ de cada matriz (probabilidad de paso de la categoría 1 a 2) fue modificado, asignándole valores dentro de cada rango permitido (de acuerdo con la ecuación 7) y dejando los demás componentes de la matriz inalterados. La Figura 4 muestra el comportamiento similar de la población del murrapal mixto bajo ambos tratamientos ante cambios dados en dicho parámetro (curvas de pendiente

CUADRO 6

Matrices de sensibilidad para las poblaciones de E. oleracea

TABLE 6

Sensitivity matrices of E. oleracea populations

$\begin{array}{cccccc}\text { Murrapal mixto testigo } & & & & \\ 0.335 & 0.031 & 0.021 & 0.009 & 0.005 & -019 \\ 1.441 & 0.135 & - & - & - & - \\ - & 0.226 & 0.148 & - & - & - \\ - & - & 0.209 & 0.087 & - & - \\ - & - & - & 0.099 & 0.062 & 0.233\end{array}$

Murrapal mixto intervenido

$\begin{array}{cccccc}0.219 & 0.031 & 0.023 & 0.010 & 0.010 & 0.017 \\ 0.907 & 0.129 & - & - & - & - \\ - & 0.237 & 0.174 & - & - & - \\ - & - & 0.234 & 0.105 & - & - \\ - & - & - & 0.125 & 0.120 & - \\ - & - & - & - & 0.145 & 0.252\end{array}$

$\begin{array}{cccccc}\text { Murrapal puro testigo } & & & & \\ 0.100 & 0.009 & 0.008 & 0.004 & 0.004 & -026 \\ 1.029 & 0.093 & - & - & - & - \\ - & 0.128 & 0.113 & - & - & - \\ - & - & 0.155 & 0.084 & 0.084 & - \\ - & - & 0.151 & 0.082 & 0.089 & 0.526\end{array}$

Murrapal puro intervenido

$\begin{array}{cccccc}0.166 & 0.022 & 0.017 & 0.013 & 0.013 & 0.015 \\ 0.903 & 0.121 & - & - & - & - \\ 1.497 & 0.200 & 0.157 & - & - & - \\ - & - & 0.194 & 0.144 & - & - \\ - & - & 0.216 & 0.160 & 0.165 & 0.248\end{array}$




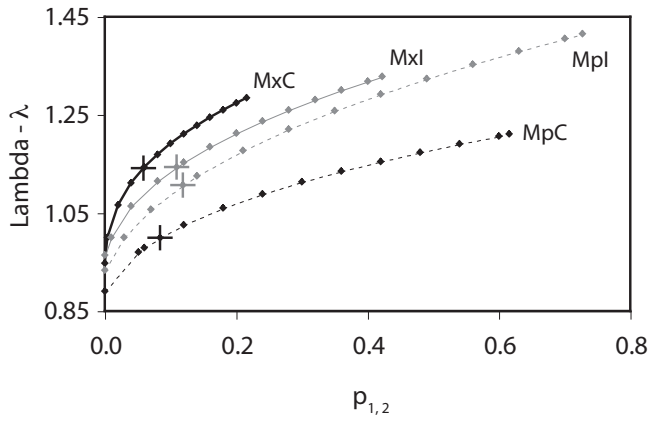

Fig. 4. Relación entre $p_{1,2} \mathrm{y}$ la tasa de crecimiento poblacional $(\lambda)$ de las poblaciones de E. oleracea (MxC: murrapal mixto control o testigo, MxI: murrapal mixto intervenido, $\mathrm{MpC}$ : murrapal puro control o testigo, $\mathrm{MpI}$ : murrapal puro intervenido, $+: p_{1,2}$ y $\lambda$ observados).

Fig. 4. Relationship between $p_{1,2}$ and population growth rate $(\lambda)$ of $E$. oleracea populations $(\mathrm{MxC}$ : mixed murrapal-control, MxI: mixed murrapal-thinned, $\mathrm{MpC}$ : pure murrapal-control, MpI: pure murrapal thinned, +: observed $p_{1,2}$ and $\left.\lambda\right)$.

comparable); sólo si esta probabilidad toma valores cercanos a 0 (0.004 y 0.009 para los tratamientos testigo e intervenido, respectivamente), las tasas de crecimiento poblacional podrían tornarse inferiores a 1. Esta figura también expone los efectos de estos cambios teóricos sobre $\lambda$ en cada tratamiento del murrapal puro, donde la curva del tratamiento intervenido presenta mayor pendiente en cualquier $p_{1,2}$, aumentando la tasa de crecimiento a una velocidad mayor que en el testigo. Si en este caso $p_{1,2}$ alcanza un valor de 0.084 , el valor estimado de $\lambda$ es 1 ; en el escenario intervenido, si $p_{1,2}$ presenta valores inferiores a 0.029 , la población entrará en declive. Asimismo, se observa la similitud entre las curvas correspondientes a ambos tratamientos intervenidos, así como en sus valores de $p_{1,2} \mathrm{y} \lambda$.

Análisis de elasticidad: La elasticidad $e_{i, j}$ cuantifica el aporte de cada $p_{i, j}$ sobre $\lambda$ (de Kroon et al. 1986). En el murrapal mixto testigo, la mayor participación la tiene $p_{1,1}$ (26.1\%), mientras que, en los demás casos, la principal contribución es de $p_{6,6}$ Esta posición aporta cerca de $20 \%$ de todos los $\lambda$, excepto en el murrapal puro testigo, donde representa casi la mitad de la tasa de crecimiento poblacional (48.3\%). La fertilidad de todas las clases de tamaño aporta $6.04-10.82 \%$ de $\operatorname{los} \lambda$, la sobrevivencia $\left(p_{i, j}, j=i\right)$ entre 56.84 y $69.66 \%$, mientras que el crecimiento $\left(p_{i, j}, j>i\right), 24.30$ $32.35 \%$. No obstante, el murrapal puro intervenido difiere de esta tendencia; allí el mayor peso lo tiene el crecimiento (50.93\%), seguido por la sobrevivencia (28.47\%) (Cuadro 7).

\section{DISCUSIÓN}

Patrones demográficos de poblaciones naturales de $E$. oleracea sometidas a diferentes niveles de inundación: Bajo condiciones sin intervención, el murrapal puro, localizado en la posición más baja del plano inundable del río Atrato y, por tanto, sometido a regímenes de inundación más prolongados y severos, así como el murrapal mixto, sujeto a regímenes de inundación menos intensos, presentaron tasas de crecimiento poblacional en equilibrio. De esta manera, los resultados permitieron rechazar la primera hipótesis planteada, donde se supone una relación directamente proporcional entre nivel y grado de inundación, y el crecimiento poblacional de la palma E. oleracea.

Lo anterior permite deducir que, si las tasas de transición observadas en ambos murrapales, sin ninguna intervención, permanecieran constantes, esta especie no cambiaría significativamente su densidad poblacional. González \& Arango (2002), utilizando técnicas matriciales propuestas por Buongiorno \& Gilles (1987), donde los ingresos periódicos fueron constantes, determinaron una tendencia creciente en el murrapal mixto y decreciente en el puro, lo cual difiere de los resultados del presente estudio. Allí, las proyecciones de la estructura poblacional mostraron que en el murrapal mixto testigo, con una ocupación en área basal promedio de $8-9 \mathrm{~m}^{2} /$ ha, se presentaría un claro crecimiento poblacional; en el murrapal puro, suponiendo el área basal de la especie de $c a$. $20 \mathrm{~m}^{2} /$ ha, la simulación indicó decrecimiento de la población. 
CUADRO 7

Matrices de elasticidad para las poblaciones de E. oleracea

CUADRO 7

Elasticity matrices of $\mathrm{E}$. oleracea populations

\begin{tabular}{|c|c|c|c|c|c|}
\hline \multicolumn{6}{|c|}{ Murrapal mixto testigo } \\
\hline 0.261 & 0.008 & 0.011 & 0.008 & 0.008 & 0.040 \\
\hline 0.074 & 0.061 & - & - & - & - \\
\hline- & 0.066 & 0.082 & - & - & - \\
\hline- & - & 0.055 & 0.032 & - & - \\
\hline- & - & - & 0.043 & 0.019 & - \\
\hline- & - & - & 0.004 & 0.036 & 0.193 \\
\hline \multicolumn{6}{|c|}{ Murrapal mixto intervenido } \\
\hline 0.132 & 0.009 & 0.013 & 0.010 & 0.015 & 0.040 \\
\hline 0.087 & 0.042 & - & - & - & - \\
\hline- & 0.078 & 0.097 & - & - & - \\
\hline- & - & 0.065 & 0.041 & - & - \\
\hline- & - & - & 0.055 & 0.066 & - \\
\hline- & - & - & - & 0.040 & 0.212 \\
\hline \multicolumn{6}{|c|}{ Murrapal puro testigo } \\
\hline 0.046 & 0.002 & 0.003 & 0.003 & 0.004 & 0.043 \\
\hline 0.054 & 0.038 & - & - & - & - \\
\hline- & 0.053 & 0.061 & - & - & - \\
\hline- & - & 0.047 & 0.037 & - & - \\
\hline- & - & 0.003 & 0.042 & 0.039 & - \\
\hline- & - & - & 0.002 & 0.041 & 0.483 \\
\hline \multicolumn{6}{|c|}{ Murrapal puro intervenido } \\
\hline 0.059 & 0.008 & 0.012 & 0.015 & 0.024 & 0.048 \\
\hline 0.097 & 0.024 & - & - & - & - \\
\hline 0.009 & 0.089 & 0.058 & - & - & - \\
\hline- & - & 0.085 & 0.059 & - & - \\
\hline- & - & 0.002 & 0.067 & 0.096 & - \\
\hline - & - & - & 0.003 & 0.045 & 0.200 \\
\hline
\end{tabular}

La mortalidad de E. oleracea, en relación con las categorías de altura, siguió la tendencia general observada en palmas clonales y no clonales: la mortalidad declina con el incremento del tamaño de la planta (van Valen 1975, Sarukhán 1978, Piñero et al. 1984, de Steven 1989, Silva et al. 1999, Barot et al. 2000, Zuidema \& Boot 2000, González \& Arango 2002). En condiciones naturales, el principal efecto de la densidad de la comunidad sobre el crecimiento en altura de E. oleracea se observó en los individuos de mayor tamaño; las probabilidades de transición de la categoría 5 a 6 fueron evidentemente mayores en el murrapal mixto, debido posiblemente a la mayor abundancia del componente arbóreo. Sin embargo, en ambas poblaciones, las tasas de crecimiento aumentaron con el tamaño de los estipes. Estos resultados concuerdan con los encontrados para Astrocaryum mexicanum (Piñero et al. 1984), donde se reportaron tasas de crecimiento más bajas para los individuos de menor tamaño; sin 
embargo, difieren con lo citado para la palma solitaria congénere Euterpe edulis (Silva et al. 1999), donde se planteó que la dependencia de la densidad sólo actúa sobre el estado de plántulas. Las anteriores observaciones reflejan alta variabilidad en la dinámica y demografía de las palmas neotropicales, incluso en casos como el aquí expuesto, donde debe existir una alta similitud en términos evolutivos dada su cercanía taxonómica.

Efecto de las cortas selectivas y la variación de la disponibilidad lumínica sobre las poblaciones de $\boldsymbol{E}$. oleracea: La extracción de estipes de E. oleracea e individuos de otras especies generó cambios en sus probabilidades de transición; los principales efectos se observaron en las categorías de altura extremas: los retoños con alturas menores a $1.3 \mathrm{~m}$ (categoría 1) prácticamente duplicaron la posibilidad de avance a la clase superior, mientras que en la categoría 5, esa tasa se redujo a la mitad. Esto sugiere la existencia de rasgos heliófitos en $E$. oleracea, con una respuesta notable a mayores ingresos de luz y reducción del desarrollo longitudinal cuando ha alcanzado los estratos dominantes del bosque (González \& Arango 2002). En ambos murrapales, el aumento de la disponibilidad de luz por efecto del aclareo del dosel produjo un incremento positivo de la tasa intrínseca de crecimiento de la población. En este sentido, la segunda hipótesis, referida al efecto positivo del incremento de la luz, se acepta.

La hipótesis arquitectural del bosque propone que el tamaño de los individuos y la competencia asimétrica por luz entre diferentes especies, donde los más grandes afectan a los más pequeños, permite explicar la coexistencia de especies (Kohyama 1993). Bajo esta óptica, la competencia por luz entre individuos de la misma especie en el murrapal mixto sería baja debido a la mayor heterogeneidad estructural. Sin embargo, tanto en este tipo de bosque como en el murrapal puro, con un dosel básicamente monoespecífico, la apertura generó mayor disponibilidad de luz para individuos de categorías inferiores, lo cual promovió la dinámica poblacional y el crecimiento en altura de algunos individuos normalmente suprimidos en el sotobosque.

Estrategias de regeneración y reclutamiento de $\boldsymbol{E}$. oleracea: La reproducción vegetativa de E. oleracea constituye una importante estrategia ecológica para su sobrevivencia. Durante el período de registro, el desarrollo de nuevos individuos genéticos o cepas (genets) fue insignificante frente a lo observado en la reproducción clonal. A través de la reproducción vegetativa, cada individuo genético se regenera conformando un conjunto de retoños, con un cono de raíces asociado. Este cono incrementa su tamaño a través del tiempo por el nacimiento de nuevos tallos, conformando un hábitat favorable para el surgimiento y sobrevivencia de nuevos retoños, los cuales se originan en la parte superior del cono de raíces. Esto apoya planteamientos que indican que, en tales casos, la reproducción vegetativa constituye una forma de cuidado postnatal (Callagham 1984, de Steven 1989). De todas formas, la probabilidad de sobrevivencia estuvo directamente asociada con las tasas de crecimiento en altura de los clones nuevos.

La reproducción vegetativa no ocurre a expensas del crecimiento o de la reproducción sexual, o de ambas (Abrahamson 1980), ya que, como sucede en Oenocarpus mapora (de Steven 1989), el meristema terminal es el responsable del crecimiento, los meristemas laterales basales de la producción de retoños y el meristema lateral superior de la floración. Por el contrario, la producción de retoños garantiza la supervivencia del individuo genético e incrementa las posibilidades de reproducción sexual (González \& Arango 2002).

Variabilidad demográfica en modelos basados en la denso-independencia: Suponer tasas de transición constantes, eje central en la mayoría de modelos determinísticos de proyección matricial, genera un escenario aplicable en el corto plazo, pero poco realista en períodos de tiempo muy prolongados. Esto ocurre principalmente porque a mayor lapso 
de la proyección, mayor dificultad de conocer el comportamiento promedio de la población. Silva et al. (1999) encontraron que los modelos lineales matriciales de crecimiento poblacional basados en estructuras de estados son adecuados para describir estructuras poblacionales y caracterizar el crecimiento, pero inadecuados cuando se requieren predicciones detalladas a largo plazo de la densidad. Resultados similares fueron reportados por González (1995) en un estudio de crecimiento de la especie arbórea Prioria copaifera (Caesalpiniaceae) en el Bajo Atrato, Pacífico colombiano.

Los análisis de sensibilidad constituyen un buen criterio para evaluar los efectos de los cambios demográficos, los que sería imposible duplicar experimentalmente. Las poblaciones de E. oleracea variaron en la dependencia del crecimiento poblacional sobre varios parámetros demográficos. El comportamiento de la población de esta especie en el murrapal mixto fue muy afectado por cambios en $p_{1,2}$, mientras que en el murrapal puro, a pesar de ser el parámetro más influyente, lo fue en menor cuantía y se destacó el rol de $p_{6,6}$ (tasas de crecimiento de la categoría 1 y de sobrevivencia de los estipes de la categoría 6, respectivamente). En el caso del murrapal mixto, el desarrollo de los retoños $\left(p_{1,2}\right)$ debió ser casi 0 para que la población tendiera al declive; en el murrapal puro se requerían ligeros aumentos en las probabilidades citadas para que la población estuviera en equilibrio $(\lambda=1)$. Particularmente, los valores ilustrados de $p_{1,2}$ muestran la magnitud de la respuesta de $\lambda$ a una unidad de cambio en esa probabilidad (cambios en la pendiente de la curva respuesta de $\lambda$ sobre el rango de valores posibles de $\left.p_{1,2}\right)$.

Resultados similares fueron reportados para la especie herbácea Dipsacus sylvestris (Caswell \& Werner 1978), donde $\lambda$ fue más afectada por alteraciones en la tasa de maduración de pequeñas rosetas que por la de individuos grandes y reproductivos. En contraste, en estudios demográficos de las especies tropicales Pentaclethra macroloba y Araucaria cunninghamii, se encontró que la población fue más sensible al crecimiento y sobrevivencia de las mayores clases de tamaño (Hartshorn 1975, Enright \& Ogden 1979). En E. oleracea, al igual que lo reportado para D. sylvestris, la mayor influencia sobre $\lambda$ fue ejercida por la tasa de crecimiento de los individuos más pequeños; de hecho, estipes de la clase 1 pueden contribuir con la producción de retoños de la cepa, disminuyendo así la importancia relativa de la sobrevivencia o la fecundidad.

Aplicaciones para el aprovechamiento y conservación de E. oleracea: Al realizar simulaciones teóricas consistentes en la alteración de un parámetro demográfico de cada matriz de transición, se encontró que los estipes o individuos de las categorías de altura 5 y 6 (8-12m y mayores de $12 \mathrm{~m}$ ) del murrapal mixto podrían ser cortados en su totalidad y las tasas de crecimiento poblacional, para ambos tratamientos, seguirían presentando valores absolutos superiores a la unidad. Claro está, la corta de estos individuos podría generar cuellos de botella en lo que a la fertilidad se refiere, ya que estas clases de tamaño son las responsables de la reproducción por semilla; sin embargo, como pudo detectarse con los análisis de elasticidad, la fertilidad, comparada con el crecimiento y la sobrevivencia, tiene el menor aporte sobre la tasa de crecimiento de la población. Además, la mayor proporción de nuevos individuos es generada mediante la propagación clonal o vegetativa.

En el murrapal puro, la tasa de crecimiento poblacional de E. oleracea bajo condiciones naturales se encontró en equilibrio; en el intervenido, la población creció, por lo que fue posible plantear su aprovechamiento. En este caso, sería posible cortar todos los individuos de la categoría de altura 6 y cerca de $30 \%$ de la categoría 5; otra opción sería cosechar hasta $40 \%$ de los de esta clase, sin intervenir alguno de la categoría 6. De esta forma, bajo ambas posibilidades de utilización, E. oleracea estaría cerca del equilibrio poblacional. 


\section{AGRADECIMIENTOS}

Los autores agradecen a CORPOURABÁ, por su apoyo mediante la orden de trabajo 020/1996: "Manejo sostenible y comunitario de la palma Euterpe oleracea (murrapo) y de los maderables asociados en la región del Medio Atrato, Colombia" y su subregional Atrato, por el apoyo logístico prestado en campo; COLCIENCIAS, a través del contrato 404/1998: "Fundamentos biológicos para el aprovechamiento sostenible de productos de las especies Euterpe oleracea y Oenocarpus bataua en la región del Medio Atrato, Colombia"; y la Dirección Nacional de Investigaciones de la Universidad Nacional de Colombia, proyecto 30803997: "Estructura y dinámica de bosques inundables en el Pacífico colombiano".

\section{RESUMEN}

La palma Euterpe oleracea es una especie dominante y promisoria en el plano inundable del río Atrato, región del Chocó, Colombia. Nosotros evaluamos la dinámica poblacional de esta especie a través de las tasas de crecimiento, la mortalidad y los patrones de reclutamiento para un período de dos años y medio. La dinámica de las tasas fue comparada entre bosques de palma mixto y puro. Estos tipos de vegetación fueron asociados con diferentes regimenes de inundación. Árboles y palmas fueron cortados en una porción de cada tipo de bosque, el resto no fue alterado. Nosotros utilizamos proyecciones matriciales para determinar las tendencias de las poblaciones. Las cortas incrementaron las probabilidades de transición de individuos pequeños pero disminuyeron las de individuos grandes, como es típico de las especies heliófitas. Las cortas también incrementaron las tasas de mortalidad en casi todas las categorías de tamaño pero no afectaron las tasas de reclutamiento. Bajo condiciones naturales, las poblaciones de E. oleracea están en equilibrio en los bosques puro y mixto. Las cortas aumentaron el crecimiento poblacional en ambos tipos de bosque, lo que sugiere el rol desempeñado por procesos denso-dependientes sobre el tamaño poblacional de esta especie.

Palabras clave: dinámica poblacional, Euterpe oleracea, demografía, modelos matriciales, sostenibilidad, Chocó Biogeográfico.

\section{REFERENCIAS}

Abrahamson, W.G. 1980. Demography and vegetative reproduction, p. 89-106. In O.T. Solbrig (ed.).
Demography and evolution in plant populations. Blackwell, Oxford, Oxfordshire, Reino Unido.

Balick, M. 1989. Native neotropical palms: a resource of global interest, p. 323-332. In G.E. Wickens, N. Haq \& P. Day (eds.). New crops for food and industry. Chapman \& Hall, Londres, Reino Unido.

Barot, S., J. Gignoux, R. Vuattoux \& S. Legendre. 2000. Demography of a savanna palm tree in Ivory Coast (Lamto): population persistence and life-history. J. Trop. Ecol. 16: 637-655.

Bernal, R. 1992. Colombian palm products, p. 158-172. In M. Plotkin \& L. Famolare (eds.). Sustainable harvest and marketing of rain forest products. Island, Washington, Columbia, EEUU.

Borgtoft, H. 1994. Mocora palm-fibers: use and management of Astrocaryum standleyanum (Arecaceae) in Ecuador. Econ. Bot. 48: 310-325.

Borgtoft, H. 1996. Production and harvest of fibers from Aphandra natalia (Palmae) in Ecuador. Forest Ecol. Manag. 80: 155-161.

Buongiorno, J. \& J.K. Gilles. 1987. Forest management and economics. MacMillan, Nueva York, Nueva York, EEUU.

Callagham, T.V. 1984. Growth and translocation in a clonal southern hemisphere sedge, Uncinia meridensis. J. Ecol. 72: 529-546.

Caswell, H. 2001. Matrix population model: construction, analysis, and interpretation. Sinauer, Sunderland, Massachussets, EEUU.

Caswell, H. \& P.A. Werner. 1978. Transient behavior and life history analysis of teasel (Dipsacus sylvestris). Ecology 59: 53-66.

Condit, R., R. Sukumar, S.P. Hubbell \& R.B. Foster. 1998. Predicting population trends from size distributions: a direct test in a tropical tree community. Am. Nat. 152: 495-509.

Connell, J.H. 1978. Diversity in tropical rain forests and coral reefs. Science 199: 1302-1310.

Coomes, D.A. \& R.B. Allen. 2007. Mortality and tree-size distributions in natural mixed-age forests. J. Ecol. 95: $27-40$.

Cortés, A. 1993. Los suelos, p. 148-155. In P. Leyva. Colombia Pacífico, Tomo 1. Fondo para la protección del Medio Ambiente "José Celestino Mutis" (FEN Colombia), Bogotá, Cundinamarca, Colombia. 
De Kroon, H., A. Plaisier, J. van Groenendael \& H. Caswell. 1986. Elasticity: the relative contribution of demographic parameters to population growth rate. Ecology 67: 1427-1431.

De Steven, D. 1989. Genet and ramet demography of Oenocarpus mapora spp. mapora, a clonal palm of Panamanian tropical moist forest. J. Ecol. 77: 579596.

Del Valle, J.I. 1996. Los bosques de guandal del delta del río Patía. Revista de la Academia Colombiana de Ciencias Exactas, Físicas y Naturales 20: 475-489.

Durán, R. \& R. Franco. 1992. Estudio demográfico de Pseudophoenix sargentii. Bulletin de l'Institut Français d'Études Andines 21: 609-621.

Enright, N. \& J. Ogden. 1979. Applications of transition matrix models in forest dynamics: Araucaria in Papua New Guinea and Nothofagus in New Zealand. Aust. J. Ecol. 4: 3-23.

Eslava, J.A. 1993. Climatología, p. 136-147. In P. Leyva. Colombia Pacífico, Tomo 1. Fondo para la protección del Medio Ambiente "José Celestino Mutis" (FEN Colombia), Bogotá, Cundinamarca, Colombia.

Espinal, L.S. 1992. Geografía ecológica de Antioquia: zonas de vida. Lealón, Medellín, Antioquia, Colombia.

Galeano, G. \& R. Bernal. 1987. Palmas del departamento de Antioquia: región occidental. Universidad Nacional de Colombia, Bogotá, Cundinamarca, Colombia.

Gonçalves, M.A. \& P.Y. Kageyama. 1994. Fenologia de floraçao e frutificação em população natural de açaizeiro (Euterpe oleracea Mart.) no estuario amazõnico. IPEF, Piracicaba, Brasil 47: 62-65.

González, H. 1995. Análisis del crecimiento diamétrico de Prioria copaifera en condiciones naturales por medio de un modelo matemático determinístico. Crónica Forestal y del Medio Ambiente 10: 101-120.

González, H. \& D.A. Arango. 2002. Efectos del clima y la densidad sobre la dinámica poblacional de Euterpe oleracea Mart. (Arecaceae) en un bosque neotropical. Crónica Forestal y del Medio Ambiente 17: 5-22.

Hartshorn, G.S. 1975. A matrix model of tree population dynamics, p. 41-51. In F. Golley \& E. Medina (eds.). Tropical ecological systems: trends in terrestrial and aquatic research. Springer, Nueva York, Nueva York, EEUU.

Henderson, A., G. Galeano \& R. Bernal. 1995. Field guide to the palms of the Americas. Princeton University, Princeton, Nueva Jersey, EEUU.
Huenneke, L.F. \& P.L. Marks. 1987. Stem dynamics of the shrub Alnus incana ssp. rugosa: transition matrix models. Ecology 68: 1234-1242.

Kohyama, T. 1993. Size-structured tree populations in gap-dynamic forest: the forest architecture hypothesis for the stable coexistence of species. J. Ecol. 81: 131-143.

Lefkovitch, L.P. 1965. The study of population growth in organisms grouped by stages. Biometrics 21: 1-18.

MacArthur, R.H. \& E.O. Wilson. 1967. The theory of island biogeography. Princeton University, Princeton, Nueva Jersey, EEUU.

Mittermeier, R.A., M. Myers, P.R. Gil \& C.G. Mittermeier. 1999. Hotspots: Earth's biologically richest and most endangered terrestrial ecoregions. CEMEX / Conservation International / Agrupación Sierra Madre, Monterrey, Nuevo León, México.

Muller-Landau, H.C., R.S. Condit, K.E. Harms, C.O. Marks, S.C. Thomas, S. Bunyavejchewin, G. Chuyong, L. Co, S. Davies, R. Foster, S. Gunatilleke, N. Gunatilleke, T. Hart, S.P. Hubbell, A. Itoh, A.R. Kassim, D. Kenfack, J.V. LaFrankie, D. Lagunzad, H.S. Lee, E. Losos, J.R. Makana, T. Ohkubo, C. Samper, R. Sukumar, I.F. Sun, N. Supardi, S. Tan, D. Thomas, J. Thompson, R. Valencia, M.I. Vallejo, G. Villa, T. Yamakura, J.K. Zimmerman, H.S. Dattaraja, S. Esufali, P. Hall, F. He, C. Hernandez, S. Kiratiprayoon, H.S. Suresh, C. Wills \& P. Ashton. 2006. Comparing tropical forest tree size distributions with the predictions of metabolic ecology and equilibrium models. Ecol. Letters 9: 589-602.

Pinard, M. 1993. Impacts of stem harvesting on populations of Iriartea deltoidea (Palmae) in an extractive reserve in Acre, Brazil. Biotropica 25: 2-14.

Pinto-Escobar, P. 1993. José Cuatrecasas y la flora y la vegetación, p. 168-181. In P. Leyva (ed.). Colombia Pacífico, Tomo 1. Fondo para la protección del Medio Ambiente "José Celestino Mutis" (FEN Colombia), Bogotá, Cundinamarca, Colombia.

Piñero, D., M. Martínez-Ramos \& J. Sarukhán. 1984. A population model of Astrocaryum mexicanum and a sensitivity analysis of its finite rate of increase. J. Ecol. 72: 991-997.

Restrepo, E. 1996. El naidí entre los "grupos negros" del Pacífico sur colombiano, p. 351-383. In J.I. del Valle y E. Restrepo (eds.). Renacientes del guandal: "grupos negros" de los ríos Satinga y Sanquianga. Proyecto Biopacífico-Universidad Nacional de Colombia, Bogotá, Cundinamarca, Colombia. 
Sarukhán, J. 1978. Studies on the demography of tropical trees, p. 163-184. In P.B. Tomlinson \& M.H. Zimmerman (eds.). Tropical trees as living systems. Cambridge University, Cambridge, Cambridgeshire, Reino Unido.

Silva, D.M., R.P. Freckleton \& A.R. Watkinson. 1999. The role of density dependence in the population dynamics of a tropical palm. Ecology 80: 2635-2650.

Silvertown, J., J.M. Franco, I. Pisanty \& A. Mendoza. 1993. Comparative plant demography-relative importance of life cycle components to the finite rate of increase in woody and herbaceous species. J. Ecol. 81: 465-476.

Svenning, J.C. \& H. Balslev. 1997. Small-scale demographic disequilibrium of Iriartea deltoidea (Arecaceae) in Amazonian Ecuador, p. 263-274. In R. Valencia \& H. Balslev (eds.). Estudios sobre diversidad y ecología de plantas. Memorias del II Congreso Ecuatoriano de Botánica. Pontificia Universidad Católica del Ecuador, Quito, Pichincha, Ecuador.

Universidad Nacional de Colombia. 1996. Diagnóstico de las existencias de la palma murrapo o naidí (Euterpe oleracea M.) y del componente arbóreo en un área del municipio de Vigía del Fuerte, región del Medio Atrato, Colombia. Universidad Nacional de Colombia, Medellín, Antioquia, Colombia.

Universidad Nacional de Colombia. 1999. Observaciones preliminares de la demografía y el crecimiento de
Euterpe oleracea en la región del Medio Atrato. Universidad Nacional de Colombia, Medellín, Antioquia, Colombia.

Universidad Nacional de Colombia. 2000. Diseño de alternativas tecnológicas sostenibles para el aprovechamiento de productos de Euterpe oleracea y Oenocarpus bataua en la región del Medio Atrato. Universidad Nacional de Colombia, Medellín, Antioquia, Colombia.

Van Groenendael, J., H. de Kroon \& H. Caswell. 1988. Projection matrices in population biology. Tree 3: 264-269.

Van Valen, L. 1975. Life, death and energy of a tree. Biotropica $7: 260-269$.

Vandermeer, J.H. \& A.H. Goldberg. 2003. Population ecology: first principles. Princeton University, Princeton, Nueva Jersey, EEUU.

Vásquez, R. \& A.H. Gentry. 1989. Use and misuse of forest-harvested fruits in the Iquitos area. Conserv. Biol. 3: 350-361.

Vormisto, J. 2002. Making and marketing chambira hammocks and bags in the village of Brillo Nuevo, northeastern Peru. Econ. Bot. 56: 27-40.

Zuidema, P.A. \& R.G.A. Boot. 2000. Demography of exploited tree species in the Bolivian Amazon. Ph. D. Thesis, Utrecht University, Utrecht, Holanda. 
